\title{
BMJ Open Rapid realist review of the role of community pharmacy in the public health response to COVID-19
}

Ian Maidment (D) , Emma Young, ${ }^{2}$ Maura MacPhee, ${ }^{3}$ Andrew Booth, ${ }^{4}$
Hadar Zaman, ${ }^{5}$ Juanita Breen, ${ }^{6}$ Andrea Hilton, ${ }^{7}$ Tony Kelly, ${ }^{8}$ Geoff Wong ${ }^{9}$

\section{ABSTRACT}

Introduction Community pharmacists and their teams have remained accessible to the public providing essential services despite immense pressures during the COVID-19 pandemic. They have successfully expanded the influenza vaccination programme and are now supporting the delivery of the COVID-19 vaccination roll-out.

Aim This rapid realist review aims to understand how community pharmacy can most effectively deliver essential and advanced services, with a focus on vaccination, during the pandemic and in the future. Method An embryonic programme theory was generated using four diverse and complementary documents along with the expertise of the project team. Academic databases, preprint services and grey literature were searched and screened for documents meeting our inclusion criteria. The data were extracted from 103 documents to develop and refine a programme theory using a realist logic of analysis. Our analysis generated 13 context-mechanismoutcome configurations explaining when, why and how community pharmacy can support public health vaccination campaigns, maintain essential services during pandemics and capitalise on opportunities for expanded, sustainable public health service roles. The views of stakeholders including pharmacy users, pharmacists, pharmacy teams and other healthcare professionals were sought throughout to refine the 13 explanatory configurations.

Results The 13 context-mechanism-outcome configurations are organised according to decision makers, community pharmacy teams and community pharmacy users as key actors. Review findings include: supporting a clear role for community pharmacies in public health; clarifying pharmacists' legal and professional liabilities; involving pharmacy teams in service specification design; providing suitable guidance, adequate compensation and resources; and leveraging accessible, convenient locations of community pharmacy.

Discussion Community pharmacy has been able to offer key services during the pandemic. Decision makers must endorse, articulate and support a clear public health role for community pharmacy. We provide key recommendations for decision makers to optimise such a role during these unprecedented times and in the future.

\section{Strengths and limitations of this study}

A diverse group of professional and public stakeholders validated our findings from the literature.

- By using a realist approach, we were able to use a broad range of data, including grey literature.

- To enable us to develop recommendations in a timely manner the focus of the review was deliberately narrow.

- The topic could have been informed by other sources of evidence in particular empirical interviews with key stakeholders.

- COVID-19 vaccination is a rapidly evolving area and this research was based on the best available evidence at the time.

\section{INTRODUCTION}

Community pharmacy teams have continued to provide essential services during the COVID-19 pandemic. They offer accessibility and medicines expertise to the public, even in challenging times. ${ }^{1-3}$ However, COVID-19 creates extra workload demands, such as medication dispensing with increases of up to $33 \%$ in prescription numbers. ${ }^{4}$ To cope with this demand community pharmacies have increased their opening hours and hired additional staff. ${ }^{4}$ Alongside this additional workload, they have managed widening coverage of the influenza vaccines programme. ${ }^{5}$

Evidence suggests that community pharmacy can successfully provide diverse vaccination services including seasonal and pandemic influenza, travel vaccinations and hepatitis B for at-risk groups, within the provisions of the UK National Health Service (NHS) or privately. ${ }^{6}$ They have successfully provided influenza vaccines as an NHS commissioned advanced service since $2015 .^{5}$ One service evaluation found that of 485 patients asked, 99\% expressed confidence in their pharmacist to provide additional vaccinations. ${ }^{7}$ Community pharmacy can also support influenza and other vaccinations to combat the 
significantly higher COVID-19-related mortality in ethnic minorities (excluding White minorities).$^{89}$

The COVID-19 pandemic has stretched NHS capacity to safely and efficiently meet public health demands. A role for community pharmacy in the national vaccination service requires an understanding of what pharmacy teams require to successfully deliver essential and advanced services during the pandemic. Such knowledge is timely, given the roll-out of COVID-19 vaccines across the community. ${ }^{10}$

Delivering a vaccination programme is a complex process and successful delivery is context dependent. A realist review helps make sense of complex situations, ${ }^{11} 12$ such as how community pharmacy can most effectively address the challenges presented by COVID-19. A rapid review can generate guidance for decision makers to assist with roll-out of COVID-19 vaccinations to community pharmacy. This rapid realist review aimed to understand how community pharmacy can most effectively deliver essential and advanced services, with a focus on vaccination, during the pandemic and in the future.

\section{METHODS}

A rapid realist review of academic and other literature, supplemented by input from key actors, was undertaken to understand how and when community pharmacy can effectively support the public health agenda during pandemics such as COVID-19. Rapid reviews aim to ensure findings are generated and disseminated in response to the urgent nature of the situation. To produce this knowledge at pace, we truncated the following review processes:

- Programme theory development was undertaken within 1 month with input from the project team.

- Searching was expedited using broad search terms and using a limited number of key data sources.

- Data analysis and context-mechanism-outcome configuration (CMOC) development focused on where the programme theory was considered most important during COVID-19.

This realist review was undertaken within 6 months (August 2020-January 2021), the protocol was published on PROSPERO ${ }^{13}$ and, where relevant, follows the Realist And Meta-narrative Evidence Syntheses: Evolving Standards (RAMESES) quality and publication standards. ${ }^{14}$

\section{Stage 1: programme theory development}

The project team met virtually to develop an embryonic programme theory using four diverse documents from an initial search representing a professional journal, ${ }^{15}$ a research journal, ${ }^{16}$ a policy document (Royal Pharmaceutical Society (RPS) $)^{17}$ and a practical influenza briefing. ${ }^{18}$ The team identified the need for (1) enabling guidance for community pharmacy (to achieve legitimisation) ${ }^{16}$; (2) practical direction for community pharmacy practices (to ensure feasibility) ${ }^{15} 16$; and (3) user assurance of appropriate, safe, feasible and timely intervention (relative advantage). ${ }^{15}$ The resultant embryonic theory, patterned on a COM-B behavioural model of capability, opportunity and motivation leading to behaviour, ${ }^{19}$ was used to inform searching and initial analyses.

\section{Stage 2: literature searching}

Searches were conducted (July-August 2020) using MEDLINE, EMBASE, CINAHL, Web of Science and Scopus for search concepts relating to Pharmacy and COVID by $\mathrm{AB}$ (see online supplemental appendix 1 for search strategy). Reference checking and citation searching of all included references on Google Scholar (using the Publish or Perish tool) were also carried out. Given the novelty of the virus, we searched the contents of preprint services and the WHO COVID Register. Grey literature searches included social media (eg, blogs, facilitated Twitter discussion (\#Cpharmchat)), community pharmacy websites and emails from relevant regulators and professional organisations (eg, RPS, Pharmaceutical Services Negotiation Committee, General Pharmaceutical Council).

Key inclusion criteria were high or middle-income countries, community pharmacy and infectious disease management (see online supplemental appendix 2). The search covered January 2003-July 2020 to include SARS, a comparable condition first identified in 2003. There were no restrictions on study designs eligible for inclusion.

\section{Stage 3: data selection and extraction (selection and coding)}

Selection and appraisal of documents followed a two-step procedure:

1. EY screened the title, abstract and keywords of potentially relevant documents against inclusion criteria. A $10 \%$ random sample was checked by two research team members ( $\mathrm{AB}$ and $\mathrm{MM}$ ) for consistency.

2. EY obtained and screened full texts of all documents meeting the eligibility criteria.

Relevant data from the included full-text documents were coded into NVivo by EY, MM and JB. Some codes came from the data (ie, inductive coding); others were derived from the programme theory (ie, deductive coding) and some were derived using retroduction (ie, by interpretation of what might be functioning as mechanisms) ${ }^{20}$ No assessment was made of the rigour of the data within included documents; however, global judgements were made of the quality of the explanations provided by the CMOCs and programme theory using the criteria of consilience, simplicity and analogy. ${ }^{21}$

\section{Stage 4: data synthesis}

The data analysis/synthesis was conducted by EY, MM and $\mathrm{JB}$ with input from the rest of the project team to develop and refine the programme theory using a realist logic of analysis. Our analysis generated 13 realist CMOCs, explaining when, why and how community pharmacy can support public health vaccination campaigns, maintain essential services during pandemics and capitalise on opportunities for expanded, sustainable public health service roles. Actor conversations generated further 
CMOCs related to care for diverse and vulnerable populations, including ethnic minorities.

Our realist logic of analysis centred on the following questions:

- Interpretation of meaning: do the contents coded by the team provide data that may be interpreted as CMOCs?

- Interpretations and judgements about CMOCs: how do the CMOCs relate to the programme theory?

- Interpretations and judgements about programme theory: how do the programme theory and its CMOCs correspond with key actor perspectives of reality?

Data to answer our questions were iteratively sought across documents. Interpretive cross-case comparison was used to identify and explain the 'success' of pandemic community pharmacy interventions delivered in different settings or to different population groups.

\section{Key stakeholders}

Key stakeholders, including community pharmacists and support staff (including representatives from large and smaller chains, sole independent pharmacies and primary care), other healthcare professionals and members of the public were consulted on four occasions. The meetings took place over Microsoft Teams and each lasted about 1 hour.

\section{Patient and public involvement}

Members of the public were drawn from The University of Sheffield's Patient and Public Involvement database and contacts of the core project team. Groups numbered between 11 and 13 members, with ages ranging from 22 to 74 years, from diverse locations and ethnicities including Black African, Black Caribbean, British Asian, British Chinese, White Irish and White British. Collectively, stakeholders provided feedback and advice on their real-world experience of working in or using community pharmacy.

\section{RESULTS}

One hundred and three documents were included in this rapid review and were coded to develop and refine our CMOCs and programme theory (Preferred Reporting Items for Systematic Reviews and Meta-Analyses diagram in online supplemental appendix 3). The final programme theory is summarised in online supplemental appendix 4-from abstract visioning to actual patient uptake of the COVID-19 vaccine. Although the programme theory is outlined in a linear fashion, steps within it are not necessarily linear and may occur simultaneously. The CMOCs are organised according to key actors, or individuals and groups with a vested interest in community pharmacy delivery of a COVID-19 vaccination programme. 'Actor' derives from sociology and is synonymous with 'stakeholder'; we privileged this term to differentiate programme theory/CMOC organisation from project stakeholder participants. ${ }^{22}$ Table 1 briefly describes the three actor groups with their respective CMOCs and corresponding steps in online supplemental appendix 4. Tables 2-4 show the final 13 CMOCs.

The following sections summarise the CMOCs related to each of the three actor groups. Illustrative examples of the supporting evidence from review documents are presented (tables 2-4).

\section{Decision makers}

Decision maker and public endorsement (CMOC 1) are essential first steps in enlisting community pharmacy for COVID-19 vaccination programmes. Regulators must ensure pharmacists have the legal scope to do so (CMOC

Table 1 Programme theory actors with corresponding CMOCs and steps

\begin{tabular}{llll}
\hline Programme theory actors & Description & CMOCs* & Steps in programme theory† \\
\hline Decision makers & $\begin{array}{l}\text { The UK government, regulatory and professional } \\
\text { bodies, the public. }\end{array}$ & $1-4$ \\
$\begin{array}{l}\text { Community pharmacists } \\
\text { and their teams }\end{array}$ & $\begin{array}{l}\text { Community pharmacists are healthcare } \\
\text { professionals registered by the General } \\
\text { Pharmaceutical Council and supported by teams } \\
\text { made up of counter assistants, dispensers } \\
\text { and registered technicians. They work in high } \\
\text { street locations, in local communities and in } \\
\text { supermarkets. Employers range from large } \\
\text { chains to small individually owned community } \\
\text { pharmacies. }\end{array}$ \\
\hline $\begin{array}{l}\text { Members of the public who use any community } \\
\text { pharmacy services including prescription } \\
\text { dispensing, minor ailment advice/treatment or } \\
\text { vaccination services. }\end{array}$ \\
\hline
\end{tabular}

*See tables 2-4.

†See online supplemental appendix 4.

CMOC, context-mechanism-outcome configuration. 
Table 2 Decision makers (CMOCs 1-4)

CMOC $1-$ support a public health role $23-27$ $6266-69$
When the government, pharmacy regulators, professional bodies and the public endorse and support a clear role for community pharmacy in public health services (C), community pharmacists will be more likely to adopt vaccination services $(0)$ because they see it as their professional role and duty (M).

'Distribution and administration of the COVID-19 vaccination programme will require concerted action across the NHS. With unique insight and expertise in medicines and the delivery of vaccination programmes, pharmacists have a clear role in contributing to the success of this programme.' (Great Britain) ${ }^{66}$

'55\% of the public have visited a pharmacy during the COVID-19 crisis...89\% of people believe pharmacies are playing an essential role in the COVID-19 crisis.' (UK National Pharmacy Association) ${ }^{27}$

'Pharmacists... have been called on to coordinate the administration of COVID-19 tests...providing ongoing COVID-19 surveillance to communities by allowing walk-in testing at community pharmacies... [This] might be more sustainable and convenient than the large-scale public screening being done as of the summer of 2020.' (USA) ${ }^{23}$

'Given the past success of community pharmacists with increasing annual seasonal influenza uptake and their accessibility, pharmacists will need to be central in administering COVID-19 vaccines in order to achieve rapid population-wide coverage.' (Canada) ${ }^{26}$

CMOC 2-clarify When pharmacy regulators and the NHS clarify community pharmacists' legal and professional legal and professional liabilities $24286670-76$ liabilities arising from the administration of a novel and potentially unlicensed COVID-19 vaccine (C), community pharmacists are more willing to give the vaccination $(0)$ because they feel reassured regarding liability $(\mathrm{M})$.

'The role of pharmacists in the COVID-19 vaccination programme must be made clear to the pharmacy profession itself. Professional and representative pharmacy bodies have an important role to play in providing the right level of information to the profession to support their roles in the vaccination programme.' (Great Britain) ${ }^{66}$

'Indemnity insurance for individual healthcare professionals needs to be amended to cover this activity and be state-funded. There also needs to be clear communication to healthcare professionals, so they clearly understand that they are covered and under which circumstances this applies.' (Great Britain) ${ }^{77}$

CMOC 3-Codevelop When COVID-19 vaccination policy and service specifications have been developed with input feasible service specifications $^{1283754}$ 78-82 administration of the vaccine (C), community pharmacies are more likely to deliver the service (O) because they believe the service specification is feasible (M).

'Pharmacists ideally want input into future policy changes before they are finalized, so that these can reflect capacity and preparedness on the ground and be publicized accurately.' (Great Britain) ${ }^{1}$

CMOC 4-issue clear, relevant and timely guidance 232428313537 $46677183-92$
When government, pharmacy regulators and professional bodies provide consistent, clear, relevant and timely guidance for the delivery of the COVID-19 vaccines (C), community pharmacies are more likely to deliver the service $(0)$, because the guidance is helpful and simplifies implementation (M).

'... it is good to see that NHSE/I have provided the information we have been waiting for to review the resources we have nationwide and decide how we can bring them to bear to help the NHS defeat this virus.' (Great Britain) ${ }^{28}$

CMOC, context-mechanism-outcome configuration; NHS, National Health Service; NHSE, National Health Service England.

2), with community pharmacy input during the development of policies and protocols (CMOC 3), so that final service specifications are flexible and doable within local settings (CMOC 4).

In England community pharmacies have government contracts and partnerships to deliver vaccinations and other essential services during emergencies, including the COVID-19 pandemic. ${ }^{23-25}$ Pharmacies in the USA and Canada have also been identified as having a substantive role in vaccine administration
(CMOC 1). ${ }^{2326}$ The idea of harnessing UK community pharmacy capacity enjoys widespread public support (CMOC 2). ${ }^{27}$ However, appropriate service delivery is hampered by unfeasible operational conditions (CMOC 3$)^{1}$; for example, medication deliveries are funded by the UK government for 'vulnerable people', but this category of service users is defined narrowly by the government and misunderstood by the public, creating unrealistic expectations of community pharmacy and generating additional work. ${ }^{1}$ 


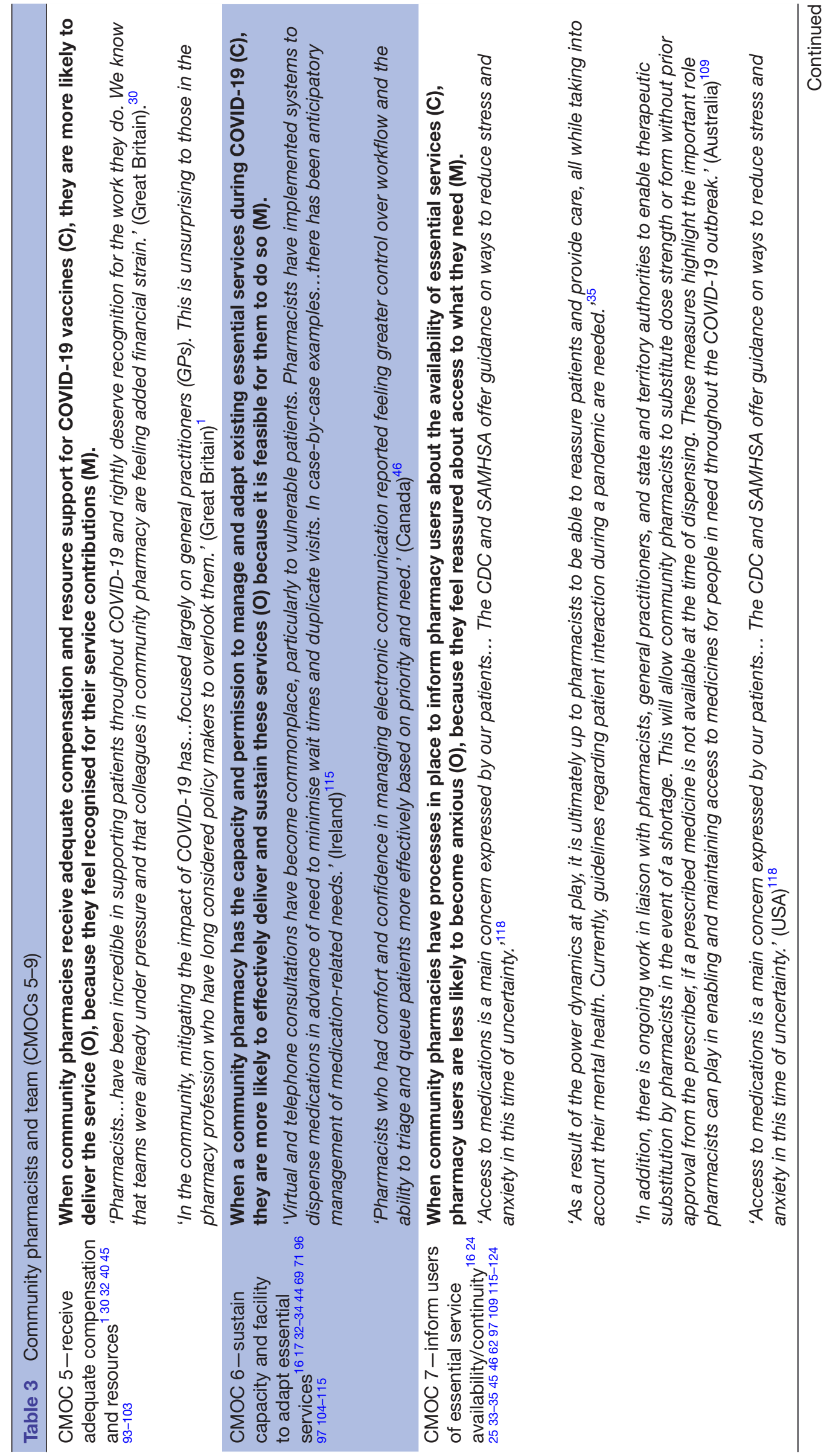




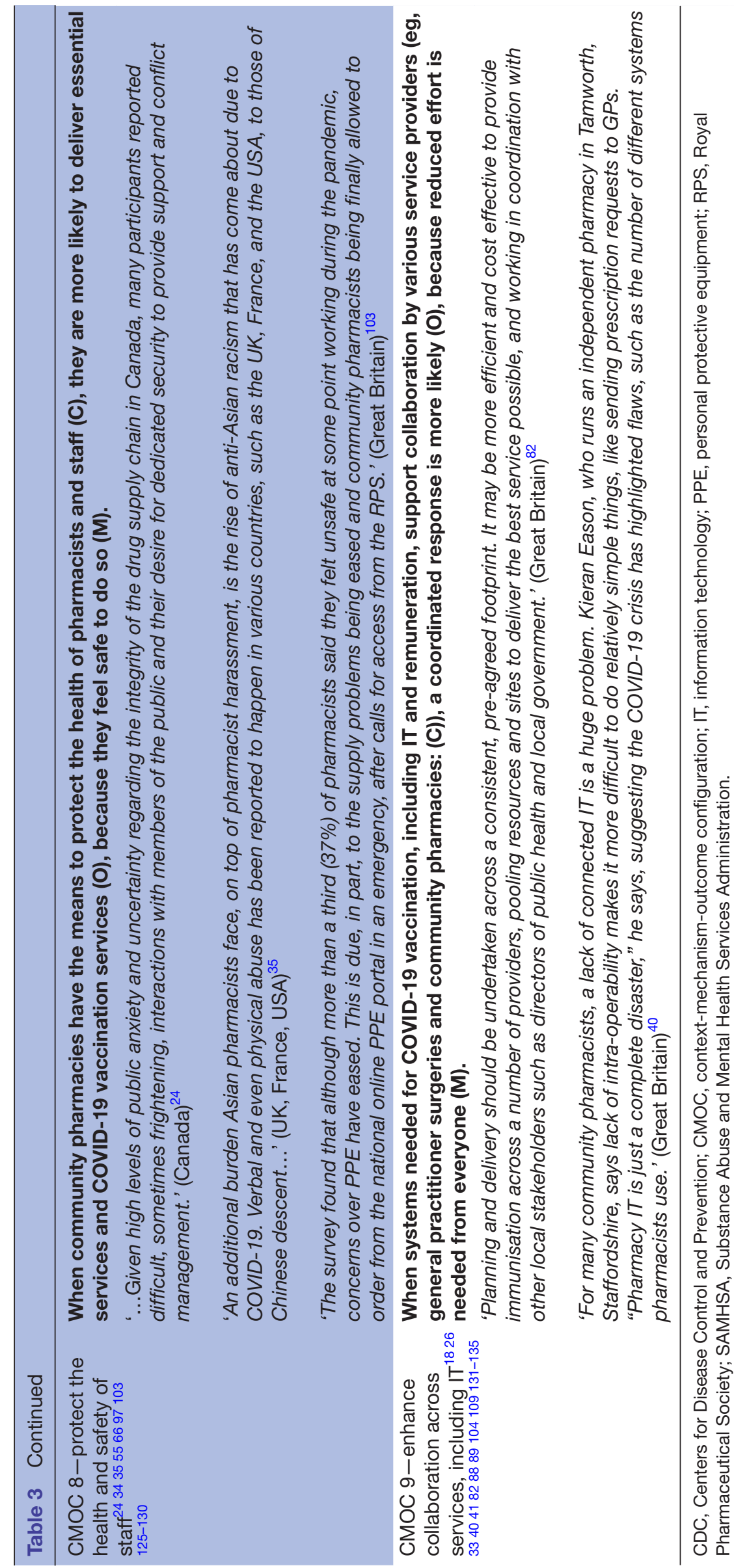


Table 4 Pharmacy users (CMOCs 10-13)

CMOC 10-trust the pharmacist as reliable information source $256185136-138$

When pharmacy users receive information about the COVID-19 vaccine from their community pharmacy $(C)$, they are more likely to get the vaccine $(O)$, because they trust community pharmacy as a source of reliable, accurate information (M).

'When patients were educated about influenza, herpes zoster, and pneumococcal vaccines as a result of a pharmacist-driven intervention in community pharmacies, they were influenced to receive the vaccination.' (USA) ${ }^{136}$

'Being able to address the public enquiries with accurate up-to-date information about the local situation and the overall infection progress is the key to build trustful relationship with them at troubled times.' (Macau) ${ }^{25}$

CMOC 11-trust the pharmacist to deliver responsive services ${ }^{71826273031}$ 3542435471748696108109 139-149

12 -access culturally sensitive services 3367104115150

When community pharmacies are trusted to make necessary service adaptations to ensure services are flexible, convenient and accessible (C), COVID-19 vaccine uptake is likely to be higher among pharmacy users $(0)$ because the service is responsive to local needs $(\mathrm{M})$.

'Pharmacists have always been the most accessible health care provider; this is especially true in the era of COVID-19.... While other professionals have closed their doors to patients, community pharmacies remained open to the public despite stricter lockdown restrictions. As highly trusted healthcare clinicians, community pharmacists play a vital role in closing the gaps that are exacerbated by the additional strain on the system and reduced access to healthcare providers.' (Canada) ${ }^{35}$

'Pharmacy flu vaccination services complement those provided by general practitioners to help improve overall coverage and vaccination rates for patients in at-risk groups. These services are highly accessed by patients from all socio demographic areas, and seem to be particularly attractive to carers, frontline healthcare workers, and those of working age.' (Great Britain) ${ }^{149}$

\section{When community pharmacies leverage their community location and community-staff} relationships (C), vulnerable populations, such as ethnic minorities, are more likely to use their services, including COVID-19 vaccination services $(0)$, because they trust their local community pharmacies to provide culturally sensitive service (M).

'Research continues to highlight that patients who are medically under-served have poorer inequitable access to health care due to them experiencing greater physical barriers to accessibility, encountering poorer patient-professional communication and are significantly disadvantaged where a service is not tailored to their unique needs or preferences.' (Great Britain) ${ }^{151}$

'I think over the years what's happened is nationally it's almost like everything has to be the same, which then doesn't work because it doesn't accommodate all the little variations...So we need to go back and in each individual pharmacy, gear it towards the population that it is meant to be meeting the needs of.' (Pharmacist caring for ethnic minority community - Great Britain) ${ }^{151}$

CMOC 13-receive private and When community pharmacies make provisions for privacy (C), pharmacy users are more likely confidential services ${ }^{7} 434583$ $152-154$ to use their services $(0)$, because they are reassured about confidentiality (M)

'If there was something not right...the first thing I would do is make an appointment with a doctor. I wouldn't do and talk to somebody over a pharmacy counter.' (Great Britain) ${ }^{45}$

'Privacy, confidentiality and dignity are all vital elements of a trusting relationship between healthcare professionals and their patients... In terms of privacy, the quality was perceived by the participants to include a confidential room that enabled private consultations.' (Great Britain) ${ }^{7}$

CMOC, context-mechanism-outcome configuration.

In contrast, when clear options for community pharmacy involvement in COVID-19 vaccination programmes were issued through the NHS (CMOC 4), the chief executive of the Association of Independent Multiple Pharmacies commented positively on members' engagement in delivering vaccines. ${ }^{28}$

\section{Community pharmacists and team}

Community pharmacies have had to manage ongoing essential services, in addition to supporting COVID-19 vaccination delivery during the pandemic (CMOC 6), including delivery of necessary medications (CMOC 7). Given fears and anxieties related to COVID-19 and changes to service delivery, community pharmacies have had to deal with inappropriate behaviours from the public, including emotional abuse and threats of physical abuse (CMOC 8). As an integral public health service, community pharmacy capacity to meet NHS needs will be enhanced through use of information technology (IT) and collaboration with other service providers (CMOC 9).

Although attention is focused on a COVID-19 vaccine, community pharmacies offer diverse essential services. In the UK and other high-income countries, community pharmacy services also provide advanced services such as vaccinations. ${ }^{29}$ Pharmacists consider it a professional 
responsibility to provide essential services during the pandemic, despite clear financial risks to themselves. ${ }^{30}$

Despite pharmacists' professional and moral obligations to provide essential services, ongoing persistent 'under-recognition' can jeopardise their ability to contribute to COVID-19 vaccinations (CMOC 5) as well as maintain a usual service. Under-recognition has been an issue in previous UK vaccination campaigns; respondents to a survey of Welsh community pharmacists after the 2016 influenza season described providing a 'mop up' service for general practitioners (GPs). ${ }^{31} 32$

Multiple required community pharmacy service adaptations have been reported by the UK Pharmaceutical Journal, including change-over in retail space to medication preparation and dispensing, and call-in shopping services for other retail items (CMOC 6). Service adaptations have been affected by hours of operation, available staff and cancellations of contracted services, such as blood pressure testing and smoking cessation support-all with financial implications for pharmacists. $^{33}$

Before COVID-19, community pharmacies globally were offering 'valued-added services' (VAS) such as drive-thru services, online ordering and communications services (eg, prescription reminders) to stay competitive. Many VAS have helped pharmacies adapt more quickly to pandemic restrictions. ${ }^{34}$

During the pandemic, ensuring access to needed medications has been a critical pharmacy service, to allay public concerns (CMOC 7). ${ }^{16}$ Potential and actual disruptions in expected services and needed supplies (eg, medications) have resulted in tensions, threats and verbal/ physical abuse by the public to community pharmacists (CMOC 8). ${ }^{33} 3536$ Safety policies, protocols and safetyrelated supplies (eg, personal protective equipment (PPE) ) must be in place to ensure community pharmacy teams' safety. ${ }^{33}$

The UK COVID-19 vaccination campaign borrows heavily from previous, successful collaborative influenza vaccination programmes using community pharmacy and GPs (CMOC 9). ${ }^{37}$ Pooling resources improves service delivery. ${ }^{38}$ For example, the UK Pharmaceutical Journal reported how a greater collaborative approach has resulted in successful influenza vaccination of home care staff and domiciliary workers during the pandemic. ${ }^{38}$

The pandemic has clearly demonstrated the importance of interoperable, connected IT systems across services. ${ }^{39}$ Getting access to reliable information is important for tracking supplies and deliveries related to the COVID-19 vaccination programme. ${ }^{40} \mathrm{~A}$ pandemic silver lining is raised awareness of IT functions for enhanced delivery of essential services (eg, medication planning, prescribing and dispensing between pharmacies and GPs) and advanced services, including vaccinations. ${ }^{40}$

\section{Pharmacy users}

Pharmacy users trust community pharmacies as a reliable source of information (CMOC 10) about vaccines, and pharmacies' local accessibility and convenience increases the likelihood of users obtaining COVID-19 vaccines through them (CMOC 11). Community pharmacy relationships with vulnerable populations in their local settings may enhance uptake of the vaccine by these groups (CMOC 12). Provision for privacy is an important user consideration. Pharmacy users expect private consultations to preserve their confidentiality (CMOC 13).

Public trust in community pharmacists is highsimilar to doctors and nurses. ${ }^{28}$ Trust between pharmacists and users can be leveraged to overcome scepticism about the COVID-19 vaccine. Providing reliable information about the disease and the vaccine, as pharmacies have done with other infections, can enhance public uptake of the COVID-19 vaccine. ${ }^{41}$ In the USA, $90 \%$ of the US population lives within 5 miles of a community pharmacy. Given their convenience and accessibility, consumers have visited their community pharmacists 12 times more frequently than their GPs. ${ }^{21}$ A UK study found that consumers who were eligible for a free influenza vaccine through their GPs were willing to pay for pharmacy service because of convenience and ease of access. ${ }^{42}$ Established, trusting relationships are especially important for providing culturally sensitive services to marginalised and vulnerable communities. ${ }^{43}$ Community pharmacists, often members of local communities, are specially positioned to understand the culturally contextual factors that impact their pharmacy users. ${ }^{44}$ Trusting relationships are founded on privacy, confidentiality and dignity, and COVID-19, public health protocols and limited space must be considered-in order to maintain vital trust among pharmacy users. ${ }^{45}$

\section{DISCUSSION}

This realist review sought to understand how community pharmacy can contribute to the public health agenda during the COVID-19 pandemic, particularly continuation of essential services and engagement in vaccination services. ${ }^{29}$ As the COVID-19 vaccination service continues to evolve, our recommendations for decision makers highlight opportunities for community pharmacy to promote safe, efficient and effective service delivery.

\section{Summary of key findings}

To optimise community pharmacy service during the pandemic, decision makers must endorse and articulate a clear role for these healthcare professionals. The public already endorses advanced roles for community pharmacy (eg, vaccinations, minor ailment scheme), but public awareness depends on what decision makers do and say. Practical decision maker measures include adequate reimbursement to help cover the cost for time, 
staff and PPE (particularly for a sustainable long-term service); legal (including indemnity), regulatory coverage for advanced roles; and clear and consistent guidance for vaccination preparation and for adaptation of essential services. When given the opportunity, the permissions and resources to do so, community pharmacies have been able to adapt quickly to continue essential services and whenever possible, to offer critical advanced services. Historically, community pharmacies have significantly increased vaccination uptake (eg, influenza vaccinations) given their accessibility and convenience and capacity to adapt to local needs for the general population and marginalised groups.

\section{Compare to other similar or related studies discussing important differences in results}

At the time of this realist review, there were no similar reviews on community pharmacy roles with respect to COVID-19 vaccinations. COVID-19 represents an unprecedented situation with limited direct evidence to guide decision-making. However, realist approaches engage with a wider evidence base, including research on past pandemics (eg, SARS), ${ }^{46-48}$ mass vaccination campaigns (eg, influenza) ${ }^{49}$ and community pharmacies' capacity pre-COVID-19 to deliver essential and advanced services. ${ }^{19} 5051$ Key factors previously include a lack of leadership, a lack of guidance and an increasing reliance on professional judgement and experience. ${ }^{46}$ Research from the UK and other economically developed countries supports the 13 CMOCs and decision maker recommendations in this review (see tables 2-5). However, limitations continue to surround the direct policy relevance of much of the community pharmacy evidence base. ${ }^{52}$

\section{Strengths and limitations}

The realist approach uses diverse data, including grey literature. This feature is especially important given a novel and rapidly evolving topic area, such as COVID-19. Multiple researchers with subject matter expertise participated in screening the literature and extracting and coding data, which maximised opportunities to discuss and debate the plausibility of the inferences made. The CMOCs were developed and refined through regular discussions within a team with varied academic and clinical backgrounds. Professional and public stakeholder consultation further refined the CMOCs.

All rapid reviews operationalise coverage versus expediency. Other sources of evidence could have informed the review; however, potential gaps were mitigated by stakeholder engagement and expertise within the team. The evidence supporting the CMOCs was based on available time for document review, and during the review period, research, directives and policy related to COVID-19 vaccination rapidly evolved. The programme theory and its CMOCs, however, are expressed in such a way that they can be further confirmed, refuted or refined in the future using additional data.

For this rapid review, given our short timeline of 6 months, we initially decided to focus on community pharmacy roles and pandemic response in middle to high-income countries. While we identified data from higher income countries such as Canada, USA, Australia and France, there was very little published from middle-income countries. As such, it is likely that our findings and recommendations are most applicable to high-income countries, although the lessons learnt from high-income countries can serve as an initial reference point for best practice for other countries.

Table 5 Recommendations for decision makers to increase community pharmacy engagement in pandemic response (for further details check: https://publications.aston.ac.uk/id/eprint/42310/1/Guidance_for_Policy_Makers_on_the_role_of_ Community_Pharmacy_in_COVID.pdf)

\section{Recommendations}

\section{Derived from CMOC}

Articulate a clear public health agenda role for community pharmacy (eg, COVID-19 testing and 1 vaccination).

Ensure pharmacy regulations for advanced roles, such as novel vaccine administration, are in place to 2 legally protect community pharmacists and their teams.

Involve local community pharmacies in policy and service specification development. 3

Provide timely guidance with sufficient details for community pharmacies to quickly adapt to local needs.

Provide adequate funding and reimbursement for community pharmacy services to deliver COVID-19 5 vaccines.

Equip community pharmacies with the necessary permissions to manage and adapt essential 6 services.

Ensure community pharmacies have the means to adequately protect the health of themselves, their 8 staff and pharmacy users.

Facilitate collaboration and coordination of COVID-19 vaccination services across providers (eg, GPs, 9 community pharmacies) and systems (eg, IT).

CMOC, context-mechanism-outcome configuration; GP, general practitioner; IT, information technology. 
Our recommendations can be viewed as 'how tos' for other countries to consider within their own cultural and healthcare environments. A number of European and North American countries have introduced legislative changes to extend the role of community pharmacy and thus reduce pressure on other parts of their healthcare systems. ${ }^{5153}$ Our findings can be used by community pharmacy and decision makers, from countries other than the UK, when mapping out similar services particularly in the context of changes in the legislation.

\section{Meaning of the study: possible explanations and implications for decision makers}

Although there are multiple actors involved in pandemic response, for brevity, recommendations (table 5) are directed towards decision makers who possess the formal authority to implement the recommendations.

As the CMOCs were refined, 'tensions' were uncovered with implications for decision makers, particularly: community pharmacists as healthcare professionals versus retailers ${ }^{51}{ }^{54-56}$; community pharmacies' capacity to remain financially viable while managing essential and advanced services ${ }^{19} 5758$; and pharmacists' capacity to provide offsite services while maintaining physical premises. ${ }^{59}$ These tensions stem from lack of awareness of community pharmacists as healthcare professionals.

Along the continuum of healthcare from community to hospital, community pharmacy is often the first point of contact for the public. Community pharmacies contribute to primary care services through essential services (eg, medication dispensing) and expanded roles (eg, vaccinations), ${ }^{29} 56$ often decreasing workload pressures on other providers, such as GPs. ${ }^{60}$ Public surveys demonstrate high levels of satisfaction with community pharmacy services, and vaccination uptake is increased when pharmacies deliver these services. ${ }^{58}$ Nevertheless, lack of public and decision maker awareness of community pharmacy primary care roles has slowed uptake and integration of these services. ${ }^{61}$ A recent UK public survey found that community pharmacies are seen as 'a medicine supply shop by $48.3 \%$ of people, as a place to purchase medicines by $22 \%$, and a place to purchase nonmedicinal products by $17.7 \%,{ }^{62}$

In Ireland and Canada, community pharmacies are an integral part of national vaccination campaigns. ${ }^{58}$ In both instances, community pharmacists participate in vaccination planning, pharmacy regulators provide clear guidance on vaccination management and vaccinations are equitably refunded through public health systems. The removal of barriers, such as economic pressures on pharmacies, has resulted in impressive national vaccine uptake, even in large countries, such as Canada. ${ }^{58}$ Globally, there is an emerging trend for governments, health insurance companies and consumers to remunerate community pharmacies for services that contribute to improved health outcomes. ${ }^{59}$

Community pharmacies tend to be defined as premises where medications are dispensed, which compounds the confusion (by the public and decision makers) of professional primary care services versus retailers. ${ }^{59}$ Instead, pharmacies should be defined with respect to actions or services that require specialised health knowledge to optimise health outcomes. For example, a retail approach to over-the-counter (OTC) medications is to permit consumers to make their own choices, similar to supermarket choices. Pharmacy input into OTC purchases could potentially decrease adverse medication interactions or unnecessary allergic reactions. ${ }^{56}$ The 'value-add' of community pharmacies, evidence-informed engagement with consumers, can decrease morbidity and mortality outcomes and increase medication regimen adherence. $^{5963}$

Although community pharmacists can provide professional services off premises (eg, immunisations in community and religious centres), they need to maintain their physical premises and staff for financial reasons. In addition, pharmacy users seek out community pharmacy services due to their accessibility and convenience, and without a physical space to engage regularly with pharmacy users, trust building between pharmacists and users is compromised. ${ }^{64}$

As evidenced by public media, many countries, including the UK, are declaring service specifications for COVID-19 vaccinations. Community pharmacists can potentially provide vaccination services in two locations. ${ }^{65}$ They can collaborate with GPs in Primary Care Networks (PCN) to support PCN vaccination sites. Alternatively, they can provide a COVID-19 vaccination service from their premises if they meet service specifications. The described tensions create difficult choices for pharmacists that can be ameliorated through the decision maker recommendations in table 5 .

\section{Unanswered questions and future research}

A future realist evaluation involving primary data collection from key actors will inform refinement of the programme theory, CMOCs and decision maker recommendations. This empirical research will address the tensions identified above. Future research will also address issues identified through the stakeholder groups, such as vaccination hesitancy, outside the scope of this review. While this work touched on unique issues for marginalised populations including ethnic minorities, those of lower socioeconomic status and those with disabilities, further exploration is needed.

We had initially focused the review on community pharmacy roles and pandemic response in middle to high-income countries, but found limited data in middleincome countries. The findings are thus more likely to be applicable to high-income countries but may serve to inform practice elsewhere. Empirical research using, for example, a realist evaluation approach should be conducted to extend our findings on the role of community pharmacy in COVID-19 vaccination programmes to middle to low-income countries. 


\section{CONCLUSION}

The COVID-19 pandemic is a worldwide health emergency. Vaccination is key to combating the pandemic. The role of community pharmacy may be both short term and long term; with a potential need for regular annual vaccines. This rapid realist review offers recommendations for decision makers to enable community pharmacy to play a key role, both during these unprecedented times and into the future.

\section{Author affiliations}

${ }^{1}$ College of Health and Life Sciences, Aston University, Birmingham, UK

${ }^{2}$ The University of Sheffield, Sheffield, UK

${ }^{3}$ The University of British Columbia, Vancouver, British Columbia, Canada

${ }^{4}$ School of Health and Related Research (ScHARR), University of Sheffield, Sheffield, UK

${ }^{5}$ University of Bradford, Bradford, UK

${ }^{6}$ College of Health and Medicine, University of Tasmania, Hobart, Tasmania, Australia ${ }^{7}$ Faculty of Health Sciences, University of Hull, Hull, UK

${ }^{8} \mathrm{NHS}$ Birmingham and Solihull Clinical Commissioning Group, Birmingham, UK

${ }^{9}$ Primary Care Health Sciences, University of Oxford, Oxford, UK

\section{Twitter lan Maidment @maidment_dr}

Acknowledgements We acknowledge the contribution from professional and member of the public key stakeholder.

Contributors IM conceived and led the project and had overall responsibility for PERISCOPE. All team members developed the embryonic programme theory. $A B$ conducted the searches. AB and GW advised on realist methods. GW also advised on primary care implications and provided methodological oversight. EY screened documents at title, abstract and full text with support from $A B$ and MM. EY, MM and $\mathrm{JB}$ coded relevant data into NVivo. EY, MM and JB conducted the data analysis/ synthesis. $\mathrm{HZ}$ and $\mathrm{AH}$ advised on pharmacy aspects and policy implications. TK led PPI. TK and $\mathrm{HZ}$ advised on implications in ethnic minorities. All team members contributed to drafting the final report for publication and approved the final draft for submission.

Funding Jointly funded by UKRI and NIHR COV0176.

Disclaimer The views expressed are those of the author(s) and not necessarily those of the NIHR, UKRI or the Department of Health and Social Care.

Competing interests GW is Deputy Chair of the NIHR HTA Prioritisation Committee: Integrated Community Health and Social Care (A) and Member of the NIHR HTA Prioritisation Committee: Integrated Community Health and Social Care (A) Methods Group. $A B$ is a member of the National Institute for Health Research Health Services and Delivery Research Funding Board, the National Institute for Health Research Evidence Synthesis Programme Advisory Group and the National Institute for Health Research School for Social Care Research Commissioning Panel.

Patient consent for publication Not required.

Provenance and peer review Not commissioned; externally peer reviewed.

Data availability statement Data are available upon reasonable request to the lead author.

Supplemental material This content has been supplied by the author(s). It has not been vetted by BMJ Publishing Group Limited (BMJ) and may not have been peer-reviewed. Any opinions or recommendations discussed are solely those of the author(s) and are not endorsed by BMJ. BMJ disclaims all liability and responsibility arising from any reliance placed on the content. Where the content includes any translated material, BMJ does not warrant the accuracy and reliability of the translations (including but not limited to local regulations, clinical guidelines, terminology, drug names and drug dosages), and is not responsible for any error and/or omissions arising from translation and adaptation or otherwise.

Open access This is an open access article distributed in accordance with the Creative Commons Attribution 4.0 Unported (CC BY 4.0) license, which permits others to copy, redistribute, remix, transform and build upon this work for any purpose, provided the original work is properly cited, a link to the licence is given, and indication of whether changes were made. See: https://creativecommons.org/ licenses/by/4.0/.
ORCID iD

Ian Maidment http://orcid.org/0000-0003-4152-9704

\section{REFERENCES}

1 Parkhurst C, Singh Purewal G, Donyai P. And COVID-19-The unsung heroes on our high streets. Journal of Patient Experience 2020;7:282-4.

2 Maidment I, Lawson S, Wong G, et al. Towards an understanding of the burdens of medication management affecting older people: the memorable realist synthesis. BMC Geriatr 2020;20:183.

3 Barry HE, Hughes CM. Managing medicines in the time of COVID-19: implications for community-dwelling people with dementia. Int J Clin Pharm 2021;43:275-9.

4 Wickware C. Pharmacies' dispensing increases by up to a third as a result of COVID-19, survey finds. Pharm J 2020.

5 Pharmaceutical Services Negotiating Committee. Flu vaccination service, 2020. Available: https://psnc.org.uk/servicescommissioning/advanced-services/flu-vaccination-service

6 Pharmaceutical Services Negotiating Committee. Vaccination services, 2020. Available: https://psnc.org.uk/servicescommissioning/locally-commissioned-services/vaccination-services

7 Rendell T. Community pharmacist-led influenza vaccination: a service evaluation. The Pharmaceutical Journal 2020

8 Public Health England. Beyond the data: understanding the impact of COVID-19 on BamE groups. London: Public Health England, 2020. https://assets.publishing.service.gov.uk/government/uploads/ system/uploads/attachment_data/file/892376/COVID_stakeholder_ engagement_synthesis_beyond_the_data.pdf

9 Patel M. We must tear down the barriers to equality for BamE communities highlighted by COVID-19. Pharmaceutical Journal 2020.

10 Robinson J. Ten things pharmacists should know about COVID-19 vaccines. The Pharmaceutical Journal 2020.

11 Wong G, Brennan N, Mattick K, et al. Interventions to improve antimicrobial presCribing of doctors in training: the impact (improving antimicrobial presCribing of doctors in training) realist review. BMJ Open 2015;5:e009059.

12 Maidment ID, Lawson S, Wong G. Health services and delivery research. medication management in older people: the memorable realist synthesis. Southampton (UK: NIHR Journals Library, 2020.

13 Maidment I, Young E, Wong G. A rapid realist review of community pharmacy support for the public health agenda during the COVID-19 pandemic and future health emergencies. PROSPERO 2020:CRD42020194078.

14 Wong G, Greenhalgh T, Westhorp G, et al. Development of methodological guidance, publication standards and training materials for realist and meta-narrative reviews: the RAMESES (realist and Meta-narrative evidence syntheses - evolving standards) project. Health Services and Delivery Research 2014;2:1-252.

15 Pharmacies across the UK close 'intermittently' to cope with increasing pressures from COVID-19. The Pharmaceutical Journal 2020.

16 Cadogan CA, Hughes CM. On the frontline against COVID-19: community pharmacists' contribution during a public health crisis. Res Social Adm Pharm 2021:17:2032-5.

17 Royal Pharmaceutical Society. Rps guidance on ethical, professional decision making in the COVID-19 pandemic. London: Royal Pharmaceutical Society, 2020. https://www.rpharms.com/ resources/pharmacy-guides/coronavirus-covid-19/coronavirusinformation-for-pharmacists/ethical-decision-making

18 Pharmaceutical Services Negotiating Committee. PSNC briefing 023/20: early guidance on the 2020/21 seasonal influenza vaccination advanced service, 2020. Available: https://psnc. org.uk/services-commissioning/psnc-briefings-services-andcommissioning/psnc-briefing-023-20-early-guidance-on-the-202021-seasonal-influenza-vaccination-advanced-service

19 Hattingh L, Sim TF, Sunderland B, et al. Successful implementation and provision of enhanced and extended pharmacy services. Res Social Adm Pharm 2020;16:464-74.

20 Greenhalgh T, Wong G, Westhorp G. Retroduction in realist evaluation. The RAMESES project, 2017. Available: http://www. ramesesproject.org/media/RAMESES_II_Retroduction.pdf

21 Wong G. Data gathering in realist reviews: looking for needles in haystacks. doing realist research. Thousand Oaks: SAGE Publications Ltd, 2018: 131-46.

22 Long N. Development sociology: actor perspectives. London: Routledge, 2003. 
23 Strand MA, Bratberg J, Eukel H, et al. Community pharmacists' contributions to disease management during the COVID-19 pandemic. Prev Chronic Dis 2020;17.

24 Gregory PAM, Austin Z. COVID-19: how did community pharmacies get through the first wave? Canadian Pharmacists Journal / Revue des Pharmaciens du Canada 2020;153:243-51.

25 Ung COL. Community pharmacist in public health emergencies: quick to action against the coronavirus 2019-nCoV outbreak. Res Social Adm Pharm 2020;16:583-6.

26 Elbeddini A, Prabaharan T, Almasalkhi S, et al. Pharmacists and COVID-19. J Pharm Policy Pract 2020;13

27 National Pharmacy Association. Public see pharmacies as key to the future of healthcare in wake of pandemic, 2020. Available: https://www.npa.co.uk/news-and-events/news-item/publicsee-pharmacies-as-key-to-the-future-of-healthcare-in-wake-ofpandemic

28 Wickware C. NHS England to choose 'selected' number of pharmacies to deliver at least 1,000 COVID-19 vaccines per week. The Pharmaceutical Journal 2020.

29 Pharmaceutical Services Negotiating Committee. Community pharmacy contractual framework: pharmaceutical services negotiating Committee, 2021. Available: https://psnc.org.uk/ contract-it/the-pharmacy-contract

30 Royal Pharmaceutical Society. All-Party pharmacy group warns of pressures on pharmacies. London: Royal Pharmaceutical Society, 2020. https://www.rpharms.com/about-us/news/details/ All-Party-Pharmacy-Group-warns-of-pressures-on-pharmacies\#: : text=The\%20All\%2DParty\%20Pharmacy\%20Group,continued\% 20 pressures\%20facing\%20community\%20pharmacies

31 NHS England and NHS Improvement coronavirus. Urgent preparing for community pharmacy to contribute to a potential COVID-19 vaccination programme. In: Service $\mathrm{NH}$, ed. London: NHS England and NHS Improvement coronavirus, 2020. https://www.england. nhs.uk/coronavirus/covid-19-vaccination-programme/primary-careguidance/preparing-for-community-pharmacy-to-contribute-to-apotential-covid-19-vaccination-programme

32 Evans AM, Wood FC, Carter B. National community pharmacy NHS influenza vaccination service in Wales: a primary care mixed methods study. British Journal of General Practice 2016;66:e248-57.

33 Stewart K, Barai R, Priarities N. How to keep your community pharmacy running during the COVID-19 pandemic, 2020. Available: https://pharmaceutical-journal.com/article/ld/how-tokeep-your-community-pharmacy-running-during-the-covid-19pandemic

34 Hussain R, Dawoud DM, Babar Z-U-D. Drive-thru pharmacy services: a way forward to combat COVID-19 pandemic. Res Social Adm Pharm 2021;17:1920-4.

35 Elbeddini A, Wen CX, Tayefehchamani Y, et al. Mental health issues impacting pharmacists during COVID-19. J Pharm Policy Pract 2020;13.

36 Quinn B. UK pharmacists facing abuse and violence during lockdown. The Guardian, 2020. Available: https://www.theguardian. com/world/2020/apr/02/uk-pharmacists-facing-abuse-andviolence-during-coronavirus-lockdown

37 NHS England and NHS Improvement coronavirus. Urgent preparing for general practice to contribute to a potential COVID-19 vaccination programme. service $N H E D$. London: NHS England and NHS Improvement coronavirus, 2020. https://www.england.nhs. uk/coronavirus/covid-19-vaccination-programme/primary-careguidance/preparing-for-gp-to-contribute-to-a-potential-covid-19vaccination-programme

38 Wickware C. Health board pilots delivery of flu vaccines by pharmacy technicians to manage increased demand. The Pharmaceutical Journal 2020.

39 Royal Pharmaceutical Society. The future of pharmacy in a sustainable NHS: key principles for transformation and growth. London: Royal Pharmaceutical Society, 2020. https://www. rpharms.com/recognition/all-our-campaigns/policy-a-z/future-ofpharmacy

40 Moore A. No going back: how the pandemic is changing community pharmacy. The Pharmaceutical Journal 2020;305.

41 Butt M. Covid-19 vaccination: logistical challenges for general practice. Practice Management 2021;31:20-1.

42 Adunlin G, Murphy PZ, Manis M. COVID-19: how can rural community pharmacies respond to the outbreak? J Rural Health 2021;37:153-155.

43 Fiore V. GP referrals under CPCS to go live November 1, NHSE confirms. Chemist and Druggist, 2020. Available: https://www. chemistanddruggist.co.uk/news/-gp-referrals-under-cpcs-livenovember-1-nhse-says
44 Robinson J. Government consults on allowing pharmacy technicians to administer COVID-19 and flu vaccines. The Pharmaceutical Journal 2020.

45 Saramunee K, Krska J, Mackridge A, et al. How to enhance public health service utilization in community pharmacy?: general public and health providers' perspectives. Res Social Adm Pharm 2014:10:272-84.

46 Austin Z, Martin JC, Gregory PAM. Pharmacy practice in times of civil crisis: The experience of SARS and "the blackout" in Ontario, Canada. Research in Social and Administrative Pharmacy 2007;3:320-35.

47 Alkhalili M, Ma J, Grenier S. Defining roles for pharmacy personnel in disaster response and emergency preparedness. Disaster Med Public Health Prep 2017;11:496-504.

48 Ford H, Dallas CE, Harris C. Examining roles pharmacists assume in disasters: a content analytic approach. Disaster Med Public Health Prep 2013;7:563-72.

49 Schwerzmann J, Graitcer SB, Jester B, et al. Evaluating the impact of pharmacies on pandemic influenza vaccine administration. Disaster Med Public Health Prep 2017;11:587-93.

50 Donald M, King-Shier K, Tsuyuki RT, et al. Patient, family physician and community pharmacist perspectives on expanded pharmacy scope of practice: a qualitative study. CMAJ Open 2017:5:E205-12.

51 Mossialos E, Courtin E, Naci H, et al. From "retailers" to health care providers: Transforming the role of community pharmacists in chronic disease management. Health Policy 2015;119:628-39.

52 Mossialos E, Naci H, Courtin E. Expanding the role of community pharmacists: policymaking in the absence of policy-relevant evidence? Health Policy 2013;111:135-48.

53 OECD. Beyond containment: health systems responses to COVID-19 in the OECD. organisation for economic co-operation and development, Paris, 2020. Available: https://read.oecdilibrary.org/view/?ref=119 119689-ud5comtf84\&title=Beyond Containment:Health_systems_responses_to_COVID-19_in_the OECD

54 Hindi AMK, Schafheutle El, Jacobs S. Community pharmacy integration within the primary care pathway for people with longterm conditions: a focus group study of patients', pharmacists' and GPs' experiences and expectations. BMC Fam Pract 2019;20:26.

55 Sum ZZ, Ow CJW. Community pharmacy response to infection control during COVID-19. A cross-sectional survey. Res Social Adm Pharm 2021;17:1845-52.

56 Ilardo ML, Speciale A. The community pharmacist: perceived barriers and patient-centered care communication. Int J Environ Res Public Health 2020;17:536.

57 Daly CJ, Quinn B, Mak A, et al. Community pharmacists perceptions of patient care services within an enhanced service network. Pharmacy 2020;8:172.

58 Ecarnot F, Crepaldi G, Juvin P, et al. Pharmacy-based interventions to increase vaccine uptake: report of a multidisciplinary stakeholders meeting. BMC Public Health 2019;19:1698.

59 Moullin JC, Sabater-Hernández D, Fernandez-Llimos F, et al. Defining professional pharmacy services in community pharmacy. Res Social Adm Pharm 2013;9:989-95.

60 NHS England. General practice forward view: NHS England, 2016. [Available from. Available: https://www.england.nhs.uk/gp/gpfv. Accessed 12/12/20

61 Hindi AMK, Schafheutle El, Jacobs S. Patient and public perspectives of community pharmacies in the United Kingdom: a systematic review. Health Expectations 2018;21:409-28.

62 Hindi AMK, Jacobs S, Schafheutle EI. Solidarity or dissonance? A systematic review of pharmacist and GP views on community pharmacy services in the UK. Health Soc Care Community 2019;27:565-98.

63 Milosavljevic A, Aspden T, Harrison J. Community pharmacist-led interventions and their impact on patients' medication adherence and other health outcomes: a systematic review. Int J Pharm Pract 2018;26:387-97.

64 Justwan F, Baumgaertner B, Carlisle JE, et al. The effect of trust and proximity on vaccine propensity. PLoS One 2019;14:e0220658.

65 Pharmaceutical Services Negotiating Committee. Preparations for a potential COVID-19 vaccination programme: pharmaceutical services negotiating Committee, 2020. Available: https://psnc.org uk/our-news/preparations-for-a-potential-covid-19-vaccinationprogramme

66 Royal Pharmaceutical Society. RPS statement on the role of pharmacists in the COVID-19 vaccination programme. London: Royal Pharmaceutical Society, 2020. https://www.rpharms.com/ Portals/O/FINAL_Statement\%20on\%20COVID-19\%20Vaccination Dec\%202020 .pdf?ver=2020-12-02-143304-017 
67 Kirkdale CL, Nebout G, Taitel M, et al. Implementation of flu vaccination in community pharmacies: understanding the barriers and enablers. Annales Pharmaceutiques Françaises 2017;75:9-16.

68 Deslandes R, Evans A, Baker S, et al. Community pharmacists at the heart of public health: a longitudinal evaluation of the community pharmacy influenza vaccination service. Res Social Adm Pharm 2020;16:497-502.

69 Sousa Pinto G, Hung M, Okoya F, et al. FIP's response to the COVID-19 pandemic: Global pharmacy rises to the challenge. Research in Social and Administrative Pharmacy 2021;17:1929-33.

70 Burson RC, Buttenheim AM, Armstrong A, et al. Community pharmacies as sites of adult vaccination: a systematic review. Hum Vaccin Immunother 2016;12:3146-59.

71 Nadeem MF, Samanta S, Mustafa F. Is the paradigm of community pharmacy practice expected to shift due to COVID-19? Res Social Adm Pharm 2021;17:2046-8.

72 Francis M, Hinchliffe A, Wales N. Vaccination services through community pharmacy. public health Wales NHS trust, Wales: NHS Wales, 2010. Available: http://www2.nphs.wales.nhs.uk: 8080/HealthServiceQDTDocs.nsf/public/CBDDC3C0BE944939 8025793B00341A6F/\$file/Vaccination\%20services\%20through\% 20community\%20pharmacy\%20v1a.pdf

73 Clews G. Pharmacists able to administer unlicensed COVID-19 vaccines following legislation changes. The Pharmaceutical Journal 2020;305. doi:10.1211/PJ.2020.20208453

74 Welsh Government, Department of Health N. I, Scottish Government. Supporting pharmacists, pharmacy technicians and pharmacy teams throughout the second COVID-19 wave, 2020. Available: https://www.pharmacyregulation.org/news/joint-lettersupporting-pharmacy-professionals-and-teams-through-secondcovid-19-wave

75 Dyer C. Covid-19: government poised to amend regulations to allow use of unlicensed vaccine. BMJ 2020;370:m3757.

76 Cooper RJ. Pestle and mortal: the demise of community pharmacy in the UK. Int J Pharm Pract 2020;28:205-6.

77 Robinson J. Health professionals delivering unlicensed vaccines need government protection, say health bodies. The Pharmaceutical Journal 2020

78 Warner JG, Portlock J, Smith J, et al. Increasing seasonal influenza vaccination uptake using community pharmacies: experience from the Isle of Wight, England. Int J Pharm Pract 2013;21:362-7.

79 Voice P, The Pharmaceutical Services Negotiating Committee, The Royal Pharmaceutical Society. Consultation response: UK influenza pandemic preparedness strategy 20112011.

80 Bauman JL. Hero clinical pharmacists and the COVID-19 pandemic: Overworked and overlooked. J Am Coll Clin Pharm 202010.1002/ jac5.1246. [Epub ahead of print: 05 May 2020].

81 Stone JK, Pate AN. The impact of COVID-19 through the eyes of a Fourth-Year pharmacy student. Am J Pharm Educ 2020;84:ajpe8146.

82 Royal College of General Practitioners. Delivering mass vaccinations during COVID-19 a logistical guide for general practice. London: Royal College of General Practitioners, 2020. https://www.Imc.org. uk/visageimages/Covid-19/RCGPMassVaccinationattimeofcovidV3 1.pdf

83 Koster ES, Philbert D, Bouvy ML. Impact of the COVID-19 epidemic on the provision of pharmaceutical care in community pharmacies. Res Social Adm Pharm 2021;17:2002-2004.

84 Bukhari N, Rasheed H, Nayyer B, et al. Pharmacists at the frontline beating the COVID-19 pandemic. J Pharm Policy Pract 2020;13.

85 Eidt M. Pharmacists on the frontline. Can Pharm J 2020;153:186-90.

86 Farrell B, Tsuyuki RT. Pharmacists step up to the challenge of COVID-19. Can Pharm J 2020;153:185.

87 Bragazzi NL, Mansour M, Bonsignore A, et al. The role of hospita and community pharmacists in the management of COVID-19: towards an expanded definition of the roles, responsibilities, and duties of the pharmacist. Pharmacy 2020;8:140.

88 Chan AHY, Rutter V, Ashiru-Oredope D, et al. Together we unite: the role of the Commonwealth in achieving universal health coverage through pharmaceutical care amidst the COVID-19 pandemic. J Pharm Policy Pract 2020;13.

89 Moore A. Pharmacists in one county say working with GP practices during the COVID-19 pandemic has been 'difficult'. The Pharmaceutical Journal 2020.

90 Hoti K, Jakupi A, Hetemi D, et al. Provision of community pharmacy services during COVID-19 pandemic: a cross sectional study of community pharmacists' experiences with preventative measures and sources of information. Int J Clin Pharm 2020;42:1197-206.

91 Chahine EB. The role of pharmacists in the fight against COVID-19. Sr Care Pharm 2020;35:286-91.
92 Jubraj B. Remote consultations: how pharmacy teams can practise them successfully: pharmaceutical-journal.com, 2020. Available: https://pharmaceutical-journal.com/article/ld/remote-consultationshow-pharmacy-teams-can-practise-them-successfully

93 Barnes E, Bullock A, Allan M, et al. Community pharmacists' opinions on skill-mix and delegation in England. Int $\mathrm{J}$ Pharm Pract 2018;26:398-406.

94 Houle SKD, Grindrod KA, Chatterley T, et al. Paying pharmacists fo patient care: a systematic review of remunerated pharmacy clinical care services. Can Pharm J 2014;147:209-32.

95 Burns C. Pharmacy staff in Scotland to receive $£ 500$ bonus in recognition of COVID-19 service. The Pharmaceutical Journal 2020

96 Merks P, Jakubowska M, Drelich E, et al. The legal extension of the role of pharmacists in light of the COVID-19 global pandemic. Res Social Adm Pharm 2021;17:1807-12.

97 Zaidi STR, Hasan SS. Personal protective practices and pharmacy services delivery by community pharmacists during COVID-19 pandemic: results from a national survey. Res Social Adm Pharm 2021;17:1832-1837.

98 Anon. Pharmacies must be at the forefront of the COVID-19 vaccination campaign. The Pharmaceutical Journal 2020;305.

99 Robinson J. England has lowest number of community pharmacies in five years, figures show. The Pharmaceutical Journal 2020.

100 Wickware C. Pharmacies to be paid 50p more per flu vaccination in 2020/2021. The Pharmaceutical Journal 2020.

101 Doyle-Price J. Pharmacies have an important role in this pandemic - but they need help to continue saving lives. The Telegraph, 2020. Available: https://www.telegraph.co.uk/global-health/science-anddisease/pharmacies-have-crucial-place-communities-need-helpcontinue

102 Robinson J. Up to $85 \%$ of community pharmacies could be in financial deficit by 2024 , economic analysis concludes. The Pharmaceutical Journal 2020.

103 Wilkinson E, Connelly D. Is pharmacy prepared for a resurgence of COVID-19? The Pharmaceutical Journal 2020;305.

104 Hayden JC, Parkin R. The challenges of COVID-19 for community pharmacists and opportunities for the future. Ir J Psychol Med 2020;37:198-203.

105 Mercadante AR, Yokota M, Hwang A, et al. Choosing evolution over extinction: integrating direct patient care services and value-based payment models into the community-based pharmacy setting. Pharmacy 2020;8:128.

106 Broughel J, Yatsyshina Y. Relax pharmacy regulations to help with COVID-19 testing and treatment. SSRN Electronic Journal 2020.

107 Pritchard RI, Huff J, Scheinberg N. Impact of regulatory changes on pharmacist-delivered telehealth during the COVID-19 pandemic. $J$ Am Pharm Assoc 2020;60:e76-9.

108 Lynch M, O'Leary AC. COVID-19 related regulatory change for pharmacists - The case for its retention post the pandemic. Res Social Adm Pharm 2021;17:1913-9.

109 Erku DA, Belachew SA, Abrha S, et al. When fear and misinformation go viral: Pharmacists' role in deterring medication misinformation during the 'infodemic' surrounding COVID-19. Res Social Adm Pharm 2021;17:1954-63.

110 Andrawis M, Carmichael J, Collins CD, et al. Improving patient care and demonstrating value during a global pandemic: recommendations from leaders of the pharmacy accountability measures work group. Am J Health Syst Pharm 2020;77:2003-5.

111 Kawaguchi-Suzuki M, Nagai N, Akonoghrere RO, et al. COVID-19 pandemic challenges and lessons learned by pharmacy educators around the globe. Am J Pharm Educ 2020;84:ajpe8197.

112 General Pharmaceutical Council. Rising to the challenge [blog], 2020. Available: https://www.pharmacyregulation.org/regulate/blog/ rising-challenge

113 Nessel J. Pharmacy technicians rise to meet patient needs during coronavirus pandemic. Pharmacy Times, 2020. Available: https:// www.pharmacytimes.com/view/pharmacy-technicians-rise-tomeet-patient-needs-during-coronavirus-pandemic

114 Green TC, Bratberg J, Finnell DS. Opioid use disorder and the COVID 19 pandemic: a call to sustain regulatory easements and further expand access to treatment. Substance Abuse 2020;41:147-9.

115 Carico R. Pharmacists continue to protect communities during COVID-19. Journal of the American Pharmacists Association 2020;60:660-1.

116 Bond C, Blenkinsopp A, Inch J. The effect of the new community pharmacy contract on the community pharmacy workforce. London: Pharmacy Practice Research Trust 2008;8:9.

117 Melton BL, Lai Z. Review of community pharmacy services: what is being performed, and where are the opportunities for improvement? Integr Pharm Res Pract 2017;6:79-89. 
118 Wilson CG, Ramage M, Fagan EB. A primary care response to COVID-19 for patients with an opioid use disorder. J Rural Health 2021;37:169-171.

119 Kostev K, Lauterbach S. Panic buying or good adherence? increased pharmacy purchases of drugs from wholesalers in the last week prior to Covid-19 lockdown. J Psychiatr Res 2020;130:19-21.

120 Bell JS, Reynolds L, Freeman C, et al. Strategies to promote access to medications during the COVID-19 pandemic. Aust $J$ Gen Pract 2020;49:530-2.

121 Romano S, Galante H, Figueira D, et al. Time-Trend analysis of medicine sales and shortages during COVID-19 outbreak: data from community pharmacies. Res Social Adm Pharm 2021;17:1876-81.

122 Gross AE, MacDougall C. Roles of the clinical pharmacist during the COVID-19 pandemic. J Am Coll Clin Pharm 2020;3:564-6.

123 Lapeyre-Mestre M, Boucher A, Daveluy A, et al. Addictovigilance contribution during COVID-19 epidemic and lockdown in France. Therapies 2020;75:343-54.

124 Alderman C. Pharmacy services and the novel coronavirus. The Senior Care Pharmacist 2020;35:146-7.

125 Hasan SS, Kow CS, Zaidi STR. Social distancing and the use of PPE by community pharmacy personnel: does evidence support these measures? Res Social Adm Pharm 2021;17:456-9.

126 Thomas CEL, Phipps DL, Ashcroft DM. When procedures meet practice in community pharmacies: qualitative insights from pharmacists and pharmacy support staff. BMJ Open 2016;6:e010 851-e51.

127 NHS England and NHS Improvement coronavirus. Standard operating procedure: COVID-19 local vaccination services deployment in community settings. London: National Health Service, 2020. https://www.england.nhs.uk/coronavirus/publication/ standard-operating-procedure-covid-19-local-vaccination-servicesdeployment-in-community-settings

128 Cabas P, Di Bella S, Giuffrè M, et al. Community pharmacists' exposure to COVID-19. Res Social Adm Pharm 2021:17:1882-7.

129 Al-Quteimat OM, Amer AM. SARS-CoV-2 outbreak: how can pharmacists help? Res Social Adm Pharm 2021;17:480-2.

130 Dawoud D. Emerging from the other end: key measures for a successful COVID-19 lockdown exit strategy and the potential contribution of pharmacists. Res Social Adm Pharm 2021;17:19501953.

131 Weir NM, Newham R, Bennie M. A literature review of human factors and ergonomics within the pharmacy dispensing process. Research in Social and Administrative Pharmacy 2020;16:637-45.

132 Maine LL. An Unparalleled spirit of collaboration amid the COVID-19 pandemic. Am J Pharm Educ 2020;84:ajpe8155-ajpe55.

133 Dowden $A$. Will serious shortage protocols help ease drug shortages? Prescriber 2020;31:29-31.

134 NHS England, NHS Improvement coronavirus. Novel coronavirus (COVID-19) standard operating procedure-Community pharmacy, 2020. Available: https://www.england. nhs. uk/wp-content/ uploads/2020/02/20200305-COVID-19-PRIMARY-CARE-SOPCOMM-PHARM-PUBLICATION-V1

135 National Pharmacy Association (NPA). Pharmacy on the NHS frontline 2020, 2020. Available: https://nhsfrontline.com/ coronavirus-stories-from-the-health-service-frontline [Accessed 17/08/2020].

136 Sparkman A, Brookhart AL, Goode J-VKR. The impact of an immunization check-up at a pharmacist-provided employee health screening. J Am Pharm Assoc 2017;57:S274-8.

137 Rodgers RM, Gammie SM, Loo RL, et al. Comparison of pharmacist and public views and experiences of community pharmacy medicines-related services in England. Patient Prefer Adherence 2016;10:1749-58.

138 Letley L, Ramsay M. Discussing the MMR vaccine with patients. The Pharmaceutical Journal 2019.

139 Kember J, Hodson K, James DH. The public's perception of the role of community pharmacists in Wales. Int $J$ Pharm Pract 2018;26:120-8.

140 Gidman W, Ward P, McGregor L. Understanding public trust in services provided by community pharmacists relative to those provided by general practitioners: a qualitative study. BMJ Open 2012;2:e000939.

141 McConeghy KW, Wing C. A national examination of pharmacybased immunization statutes and their association with influenza vaccinations and preventive health. Vaccine 2016;34:3463-8.

142 Cheong MWL, Brock T, Karwa R. COVID-19 and clinical pharmacy worldwide - a wake up call and a call to action. JACCP JAm Coll Clin Pharm 2020;3:860-3.

143 Aruru M, Truong H-A, Clark S. Pharmacy emergency preparedness and response (pepR): a proposed framework for expanding pharmacy professionals' roles and contributions to emergency preparedness and response during the COVID-19 pandemic and beyond. Res Social Adm Pharm 2021;17:1967-77.

144 Robinson J. Public health England to review guidance against the use of rapid COVID-19 tests in community pharmacies. The Pharmaceutical Journal 2020;305.

145 Buchan SA, Rosella LC, Finkelstein M, et al. Impact of pharmacist administration of influenza vaccines on uptake in Canada. CMAJ 2017;189:E146-52.

146 Calo WA, Shah PD, Gilkey MB, et al. Implementing pharmacylocated HPV vaccination: findings from pilot projects in five U.S. states. Hum Vaccin Immunother 2019;15:1831-8.

147 Anderson C, Thornley T. "It's easier in pharmacy": why some patients prefer to pay for flu jabs rather than use the National Health Service. BMC Health Serv Res 2014;14.

148 Baroy J, Chung D, Frisch R, et al. The impact of pharmacist immunization programs on adult immunization rates: a systematic review and meta-analysis. J Am Pharm Assoc 2016;56:418-26.

149 Anderson C, Thornley T. Who uses pharmacy for flu vaccinations? population profiling through a UK pharmacy chain. Int J Clin Pharm 2016;38:218-22.

150 Pharmacy Guild of Australia. 93 per cent of Aussies recognise benefits of flu shot in pharmacy-Pharmacy Guild of Australia [blog]: Pharmacy Guild of Australia 2017, 2017. Available: https://www. guild.org.au/news-events/news/2017/93-per-cent-of-aussiesrecognise-benefits-of-flu-shot-in-pharmacy

151 Latif A, Tariq S, Abbasi N, et al. Giving voice to the medically Under-Served: a qualitative Co-Production approach to explore patient medicine experiences and improve services to Marginalized communities. Pharmacy 2018;6:13.

152 Anderson C, Blenkinsopp A, Armstrong M. Feedback from community pharmacy users on the contribution of community pharmacy to improving the public's health: a systematic review of the peer reviewed and non-peer reviewed literature 1990-2002. Health Expect 2004;7:191-202.

153 Eades CE, Ferguson JS, O'Carroll RE. Public health in community pharmacy: a systematic review of pharmacist and consumer views. BMC Public Health 2011;11:582-82.

154 Hassell K, Rogers A, Noyce P. Community pharmacy as a primary health and self-care resource: a framework for understanding pharmacy utilization. Health Soc Care Community 2000;8:40-9. 